\title{
Umbilical Nodule and Hemorrhagic Ascites of Endometriosis Origin: A Clinical Case Report
}

\author{
Modia O'yandjo A ${ }^{1,2}$, Bosenge Nguma JD² and Kadima Ntokamunda J3* \\ ${ }^{1}$ Faculty of Medicine and Pharmacy, Department of Gynecology and Obstetrics, University of Kisangani, DR Congo \\ ${ }^{2}$ Clinique des Anges Kisangani, Kisangani, DR Congo \\ ${ }^{3}$ Department of Clinical Pharmacology, University of Rwanda, Rwanda
}

${ }^{*}$ Corresponding author: Kadima Ntokamunda J, Department of Clinical Pharmacology, University of Rwanda, Rwanda, Tel: (250)788422422; Email: kadima48@yahoo.com

Rec date: December 27, 2017; Acc date: February 19, 2018; Pub date: February 22, 2018

Citation: O'yandjo AM, Bosenge NJD, Kadima NJ (2018) Umbilical Nodule and Hemorrhagic Ascites of Endometriosis Origin: A Clinical Case Report. Gynecol Obstet Case Rep Vol.4:No.1:62.

\section{Abstract}

Endometriosis affects $5 \%-10 \%$ of women in reproductive age and is usually localized in the pelvic and abdominal organs resulting in chronic pain, deep dyspareunia and infertility. Exceptionally, superficial endometriosis can lead to hemorrhagic abdominal effusion. Umbilical localization is considered a rare presentation, especially in its primary form. As there are many atypical cases, the differential diagnosis between endometriosis and other soft tissue tumors can be quite difficult. We report a historical case associating primary umbilical endometriosis and hemorrhagic ascites and discuss the probable pathogenic hypotheses and difficulties related to the diagnosis.

Keywords: Pelvic pain; Umbilical endometriosis; Hemorrhagic ascites; Surgery; GnRH treatment

\section{Introduction}

Endometriosis is defined as the presence of functional endometrial tissue in a location other than the uterine cavity $[1,2]$. The preferential site is abdominopelvic. Under these conditions, endometriosis is usually accompanied by dysmenorrheal, chronic pelvic pain, deep dyspareunia, and infertility. Exceptionally, it can lead to abdominopelvic effusion. Extra abdominal forms account for about $15 \%$ of cases [3] and have been described in almost all tissues (skin, surgical scars, hernia sac, lungs, pleura, etc.) [4,5]. The umbilical location is often secondary, occurring after laparoscopy or any other surgical procedure involving the umbilicus. The primary umbilical endometriosis constitutes a rare entity [6,7] and often poses a problem of differential diagnosis with melanoma, umbilical metastasis, pyogenic granuloma, umbilical hernia, etc. [8]. Several theories have been advanced to clarify its pathogenesis, but none of them could be completely exhaustive. We report here a historical case observed at a tertiary hospital in Kisangani city (DR
Congo), associating a primary umbilical endometriosis and a hemorrhagic ascites while remembering the difficulties related to the diagnosis and the probable pathogenic hypotheses.

\section{Case Presentation}

A 31-year-old nulliparous black woman was transferred to our service in August 2014 for abdominal pain. The history indicated she had her menarche at the age of 10 . At the age of 18 , she was suffering from dysmenorrheal. She had consulted 3 years ago for infertility and no obvious cause was objectified. One year ago, she had to stop working because of the aggravation of pelvic pain. At the same time, she noticed an increase in the volume of the umbilicus, which became painful. Within 10 months, the abdomen ballooned and the diagnosis of uterine fibromyoma and moderate ascites was made. A few months later her situation worsened, and she was then transferred to a department of internal medicine. The ascites puncture drained 1.5 litres of citric fluid rich in polynuclear lymphocytes, with no atypical cell. The culture on different media was sterile, but the patient was defaulted on antituberculous treatment and diuretics. With this treatment, the patient dehydrated and on the verge of cardiac decompensation she went see a cardiologist. The cardiologist excluded cardiac involvement, suspected Meiggs syndrome and transferred her to gynecology-obstetrics unit at CAKIS (Clinique des Anges Kisangani).

At admission, her last menstrual period went back 7 days before with 1 day in duration. The patient was dyspnetic; the abdomen was over-pronounced, completely matt and surmounted by a blistered, trilobed navel containing a moderately sensitive nodule (Figure 1). With vaginal touch, the uterus was increased in volume, with an irregular surface riddled with firm nodules. On ultrasound, there was a large multicolored ascites containing fine echoes regularly distributed. The umbilicus contained a rounded, heterogeneous, mixed image made of hypoechoic areas with fine irregular echoes. The uterus was dotted with hypoechoic images of a myomatous nature. 


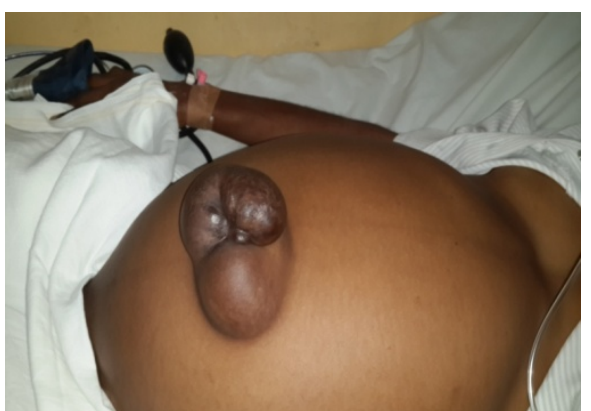

Figure 1 Abdomen ballooned; umbilical swollen and skinned.

Laparotomy allowed removing 5 liters of hemorrhagic ascites and performing myomectomy. The ovaries were normal in appearance. Several endometriosis spots were found on the parietal peritoneum. In the umbilicus there was a cyst containing chocolate blood (Figures 2 and 3). We ablated the cyst with some part of the navel. Postoperatively, the biopsy examination confirmed the diagnosis of endometriosis and the patient was put on a gonadotrophin releasing hormone $(\mathrm{GnRH})$ analogue with disappearance of symptoms and a good evolution within two years.

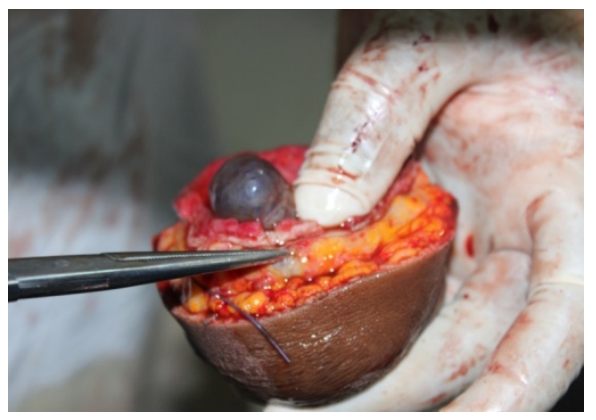

Figure 2 Endometriosis cyst fleeing into the umbilicus.

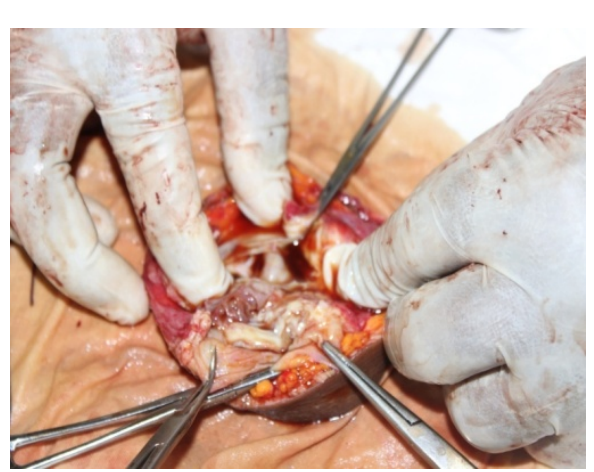

Figure 3 Dissection of the cyst showing a chocolate content.

\section{Discussion}

Endometriosis affects $5 \%-10 \%$ of women with genital activity [1,3], 38\% (20\%-40\%) of infertile women [9] and is most likely to affect women aged $30-40$ years [4]. Direct causes are not clear on these days. Several reproductive factors have been consistently associated with the risk of endometriosis, suggesting a significant impact of hormonal variations on the risk to its occurrence. For example, age at menarche [10] and hypomenorrhea [11] are associated with increased risk, whereas parity [12] and recent use of oral contraceptives [13] would be associated with decreased risk. Indeed, some of these factors were found in our patient (nulliparity, menarche at the age of 10 years and hypomenorrhea). The notions of hereditary factor and alteration of the immune system were also evocated [14].

Umbilical endometriosis represents $0.4 \%-4 \%$ of ectopic localizations of the endometrium and about $30 \%-40 \%$ of cutaneous endometrioses [3]. It is often secondary, due to iatrogenic dissemination and umbilical implantation of endometrial cells by surgical manipulation. The peculiarity of our case is that the patient had a primary endometriosis, spontaneously occurring outside any surgical procedure. It is a rare entity, described in $0.5 \%-1 \%$ of extra pelvic locations [6]. It usually presents as a dark or bluish nodule, swollen or not, whose size usually varies with the menstrual cycle; and is often accompanied by cyclic pain. However, there may be atypical cases so that the differential diagnosis between endometriosis and other soft tissue tumors becomes quite difficult.

The pathogenesis of primary umbilical endometriosis has not yet been fully elucidated, but four theories have been raised [3,15-17]. The first is the metastatic theory of Ivanov and Meyer suggesting that under the influence of infectious, hormonal, toxic and traumatic factors, cells derived from the coelomic epithelium retain their embryonic potentials and undergo metaplasia in endometrial cells. The second is the Sampson reflux theory corresponding to a migration of endometrial flaps by periodic tubal regurgitation of menstrual blood or by surgical contamination, and their ectopic implantation. Thirdly, the metastatic theory of Alban tries to explain the extra-genital locations by venous or lymphatic migration of endometrial cells that are grafted electively on scar obstacles or ganglionic relays particularly on the lymphatic network of the umbilicus. Finally, Lavender describes a mixed theory that menstrual regurgitations induce metaplasia of the cells of the coelomic epithelium into endometrial cells.

The pathogenesis of endometriotic hemorrhagic ascites remains speculative. It is thought to be caused by the rupture of an endometrioma ( $65 \%$ of cases) or by the exudation of generalized pelvic endometriosis. Rupture of the endometrioma is accompanied by acute pain and fever. Laparotomy causes less than $500 \mathrm{~mL}$ of effusion fluid to be seen and the area or scar of endometrial rupture [18]. Superficial endometriosis, considered a low-grade inflammation, may also be associated with a slight increase in peritoneal fluid, but not necessarily with ascites [19]. Bernstein et al. [20] had suggested that blood and endometrial 
cells that spread into the peritoneal cavity from superficial endometriotic lesions could irritate and stimulate the peritoneum, resulting in ascites. Another contributing factor is obstruction of the lymphatics in the diaphragm by extensive peritoneal endometriosis [21].

\section{Conclusion}

This case reported the association of rare primary endometriosis (umbilical) and exceptional symptomatology of pelvic endometriosis (hemorrhagic ascites). In front of an umbilical nodule, it would be advisable to think of the endometriosis especially when it adds a symptomatology of cyclical nature. The pathophysiology of endometriosisassociated hemorrhagic ascites, as well as that of primary umbilical endometriosis, is not yet clearly defined; all the theories evoked remain speculative and can be associated.

\section{Informed Consent}

The patient had consented to the publication of this case report accompanied by images.

FundingNo funding

\section{Competing and Conflicting Interests}

The authors do not declare any conflict of interest.

\section{Contributions of the Authors}

Prof Modia O'yandjo and Dr Bosenge Nguma contributed to the care of the patient and the design of the manuscript. Prof. Kadima analyzed the case and corrected the final manuscript. All three authors read and approved the final manuscript.

\section{References}

1. Parasar P, Pinar Ozcan P, Terry KL (2017) Endometriosis: Epidemiology, diagnosis and clinical management. Curr Obstet Gynecol Rep 6: 34-41.

2. Giudice LC, Kao LC (2004) Endometriosis. Lancet 364: 1789-1799.

3. Alessandro $F$, Antonio $P$, Alessandra $M$, Giampiero $C$, Alberto $P$ (2013) Primary umbilical endometriosis. Case report and discussion on management options. Int J Surg Case Rep 4: 1145-1148.

4. Dessy LA, Buccheri EM, Stefano C, Gagliardi DN, Onesti MG (2008) Umbilical endometriosis, our experience. In Vivo 22: 811-816.

5. Mistrangelo $M$, Gilbo $N$, Cassoni $P$, Micalef $S$, Faletti R, et al. (2014) Surgical scar endometriosis. Surg Today 44: 767-772.
6. Wanyonyi SZ, Gichere IH, Chege J (2012) Primary umbilical endometriosis: A case report. JOGECA 24: 25-27.

7. Paramythiotis D, Stavrou G, Panidis S, Panagiotou D, Chatzopoulos K, et al. (2014) Concurrent appendiceal and umbilical endometriosis: a case report and review of the literature. J Med Case Reports 8:258-262.

8. Jaime Thais J, Jaime Titiana J, Ormiga P, Leal F, Nogueira OM, et al. (2013) Nilton Umbilical endometriosis: report of a case and its dermoscopic features. An Bras Dermatol 88: 121-124.

9. Neha A, Arulselvi S (2010) Endometriosis- Morphology, clinical presentations and molecular pathology. I f Lab Physicians 2: 1-11.

10. Matalliotakis I, Cakmak H, Fragouli Y, Goumenou A, Mahutte N, et al. (2008) Epidemiological characteristics in women with and without endometriosis in the Yale series. Arch Gynecol Obstet 277: 389-393.

11. Darrow SL, Vena JE, Batt RE, Zielezny MA, Michalek AM, et al. (1993) Menstrual cycle characteristics and the risk of endometriosis. Epidemiology 4: 135-142.

12. Missmer S, Hankinson S, Spiegelman D, Barbieri R, Malspeis S, et al. (2004) Reproductive history and endometriosis among premenopausal women. Obstet Gynecol 104: 965-974.

13. Vercellini P, Eskenazi B, Consonni D, Somigliana E, Parazzini F, et al. (2011) Oral contraceptives and risk of endometriosis: a systematic review and meta-analysis. Hum Reprod Update 17: 159-170.

14. Randriamahavonjy R, Randriambololona DMA, Fenomanana MS, Rakotoratsimba HN (2015) A case of endometriosis in menopausal women (Un cas d'endométriose ombilicale chez une femme ménopausée in French). JMGO 1: 17-18.

15. Zrara I, Choho A, Jastimi S, Al Bouzidi A, Rimani M, et al. (2001) Umbilical endometriosis: A case report. (endométriose ombilicale in french). Médecine du Maghreb 89: 25-26.

16. Batt RE, Yeh J (2013) Mullerianosis: four developmental (embryonic) mullerian diseases. Reprod Sci 20: 1030-1037.

17. Matsuura K, Ohtake H, Katabuchi H, Okamura H (1999) Coelomic metaplasia theory of endometriosis: evidence from in vivo studies and an in vitro experimental model. Gynaecol Obstet Invest 47: 18-20.

18. Atabekoglu C, Sonmezer M, Dunder I (2002) Elevated serum CA 125 and CA 19-9 due to spontaneous rupture of endometrioma. Eur J Obstet Gynecol Reprod Biol 105: 75-76.

19. Ussia A, Betsas G, Corona R, De Cicco C, Koninckx PR (2008) Pathophysiology of cyclic hemorrhagic ascites and endometriosis. J Minimally Invasive Gynecol 15: 677-681.

20. Bernstein JS, Brenner PJ (1961) Massive ascites due to endometriosis. Am J Dig Dis 6: 1-7.

21. London S, Parmley T (1993) Endometriosis and ascites. South Med J 86: 1173-1175. 